\title{
Magnetosheath and solar wind turbulence processes features identification
}

\author{
A.S. Prokhorenkov ${ }^{1 *}$, L. V. Kozak ${ }^{1}$, A. T. Y. Lui ${ }^{2}$ \\ ${ }^{1}$ Taras Shevchenko National University of Kyiv, Glushkova ave. 4, 03127, Kyiv, Ukraine \\ ${ }^{2}$ Johns Hopkins University Applied Physics Laboratory, Laurel MD, USA
}

\begin{abstract}
A different kind of analysis should be applied to a turbulent process, than that applied to a nondisturbed medium. In this work we present the result of extended self-similarity analysis (ESS) by comparing between different turbulent models: Kolmogorov K41 model, She-Leveque (isotropic log-Poisson model) of order 3; Iroshnikov-Kraichnan model and Politano-Pouquet model of order 4. Two regimes were observed for large and small timescales: the Gaussian distribution was used for small timescales for magnetic field fluctuation probability distribution function (PDF), and the Lèvy distribution was used for large-scale timescale non-Gaussian distributions. The intersection of two asymptotes corresponds to approximately $1 \mathrm{~s}$, which agrees with the ion-cyclotron period.
\end{abstract}

Key words: magnetopause, cusp, and boundary layers; solar wind-magnetosphere interactions; nonlinear phenomena

\section{INTRODUCTION}

In the process of interaction of the solar wind with magnetosphere, we are not dealing with a sequence of additive reactions of the magnetic layer to disturbances in solar wind, but rather with a complex large-scale nonlinear system, in which a "catastrophic" restructuring of the current and magnetic topology takes place. The result is a complex turbulized environment, in which perturbations of MHDparameters of large amplitude are represented in a process with a probability significantly higher than that assumed by Gaussian distribution (we have heterogeneity (intermittence) of turbulent processes; an alternation of quasi-laminar and turbulent forms of movement).

Theoretical consideration shows that an attribute of intermittency of turbulence is associated with hidden statistical symmetries (symmetries of scale invariance) of dynamic equations describing motion [3]. Random perturbations of speed and other parameters of the turbulent flow with intermittence have non-Gaussian statistics $[1,8]$. Thus, dynamics of this process will be described by a power distribution law and multiscality (spectrum of characteristic scales). It is currently impossible to solve the problem of turbulent plasma dynamics (in threedimensional geometry) analytically or numerically, and to determine the type of turbulence with necessary details on a large time scale. Therefore, we need to determine statistical properties of turbulence from experiment and compare the results with existing models.

*andrew. prokhorenkov@gmail.com

A.S. Prokhorenkov, L. V. Kozak, A.T. Y.Lui, 2015
It should be noted that despite the fact that powerful analytical methods are developed in the theory of turbulence, modern analytical models still do not achieve the same degree of detail and accuracy as semi-empirical cascade models, based on statistical methods $[1,5,6,12]$. This is especially true for a description of turbulence with intermittence. As intermittence is not only the result of turbulent "activity", but also turbulent "activity" itself is distributed unevenly.

\section{METHODS AND APPROACHES}

For the data series $X(t)$ the evolution of maximum of PDF for investigated parameters $P_{t}(0)$ with a time scale change $\tau$ can be approximated by a power law $[1,4,7]$ :

$$
P_{\tau}(0) \sim \tau^{-s},
$$

where for Gaussian distribution the exponent is $s \sim$ 0.5 and for non-Gaussian distribution it is $s>0.5$.

The more the $s$ value differs from 0.5 , the longer the scales on which correlations in the system and reorganization processes are present.

For turbulence with intermittence, the likelihood of significant fluctuations on the wings of the distribution will be high due to excess energy of the large-scale disturbances, generated by the source.

It should be noted that examination of the probability's density is equivalent to a sequence analysis (in principle, infinite) of statistical moments. Herewith, due to normalization condition, zero-order moment is equal to one; the moment of the first order, 
also called a mathematical expectation, gives the average value of the quantity. For moments of the second and higher orders, central moments are usually used, calculated on average values. (A central moment of the second order is dispersion).

Evolution of the maximum function of density distribution with time of fluctuations' probability of MHD-parameters indicates the presence of homogeneous or heterogeneous turbulent processes.

For specification of the type of turbulent processes, an analysis of statistical moments (structural functions) of high orders has been performed.

While analysing fluctuations variable in time, a structural function $S_{q}$ of MHD-parameters $X(t)$ of the order $q$ is determined on the scale $\tau$, as a statistical average over the ensemble of relations [5]: $S_{q}(\tau)=\left\langle|X(t+\tau)-X(t)|^{q}\right\rangle \sim \tau^{\zeta(q)}$.

To analyse moments of the distribution function, a determining factor is the availability of enhanced self-similarity property (extended selfsimilarity, ESS). This phenomenon was discovered experimentally in the study of small-scale hydrodynamic turbulence in the wind tunnel, and led to development of generalized self-similarity criteria for any pair of structural features:

$$
S_{q}(l) \sim S_{p}(l)^{\frac{\zeta(q)}{\zeta(p)}} .
$$

It is assumed that such self-similarity is a manifestation of hidden statistical symmetries.

Accordingly, during analysis, structural functions are plotted on a double-logarithmic scale. Herewith, linear plots appear in inertial interval, declination of which gives the value of power indexes $\zeta(q)$.

Using the attribute of extended self-similarity, we can find with a fairly good accuracy $\zeta(q)$ and assess the type of turbulent processes.

For Kolmogorov's isotropic turbulence K41 $\zeta(q)=q / 3$, and for the two-dimensional turbulence Iroshnikov-Kraichnan model $(\mathrm{IK})-\zeta(q)=q / 4$ $[5,10,11,14]$.

It should be added that the IK model is used to explain MHD-turbulence in the presence of strong anisotropy of processes, induced by magnetic fields. Herewith, turbulent disturbances are small in amplitude and spread along the middle field (Alfvén turbulence), while Kolmogorov assumptions are valid, if the magnetic field fluctuations dominate over external magnetic field.

Nonlinear functional dependence $\zeta(q)$ on the order of the moment $q$ for experimental data is the result of intermittency of the processes.

To interpret the nonlinear dependence $\zeta(q)$, a range of models is used: lognormal, $\beta$-model, logPoisson, and others [3].

As part of the work, the comparison of results of research with log-Poisson turbulence model and Pulitano-Pouquet model was made. For log-Poisson model $\zeta(q)$ is determined by a correlation [2]:

$$
\zeta(q)=(1-\Delta) \frac{q}{3}+\frac{\Delta}{1-\beta}\left[1-\beta^{\frac{q}{3}}\right],
$$

where $\beta$ and $\Delta$ are parameters that characterize the intermittence and singularity of dissipative processes respectively. It is important to note that by varying parameters $\Delta$ and $\beta$ we can get a power index for different types of models. So, for three-dimensional isotropic turbulence, in which the logarithm of energy dissipation is described by Poisson distribution $\Delta=\beta=2 / 3$ are She and Leveque (SHL) parameters [13].

Politano and Pouquet [12] generalized SheLeveque model for a case of existence of twodimensional dissipative structures. Herewith, the dependence $\zeta(q)$ has a form of [12]:

$$
\zeta(q)=q / 8+1-(1 / 2)^{q / 4} .
$$

\section{SATELLITES USED FOR MEASUREMENT}

To study turbulent properties of the environment, ferroprobe measurements of magnetic field fluctuations of the spacecraft C2 of the "Cluster-2" mission were used.

Regions of the spacecraft's passage through the magnetic layer (one of the most perturbed regions of near-Earth space) were analysed. Herewith, KA was moving from the solar wind (SW) plasma and was consistently passing the foreshock region (FSH), the shock wave (BS), the postshock region (PSH), the magnetic layer (magnetosheath, MSH), and entering magnetopause (MP) region.

Examples of satellite measurements of the magnetic field fluctuations with discreteness of $22.5 \mathrm{~Hz}$ are shown in Fig. 1.

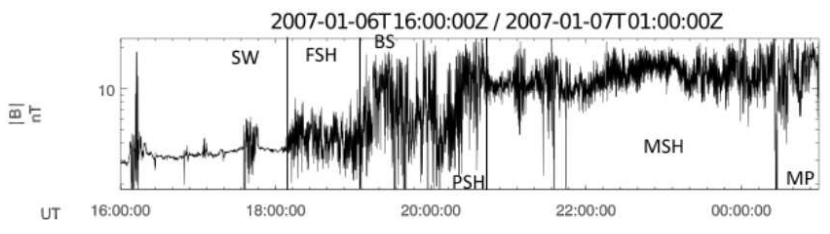

Fig. 1: Magnetic field fluctuations in different regions from the $\mathrm{C} 2$ satellite.

From Figure 1 it is evident that fluctuations in the magnetic layer differ significantly from the level of fluctuations in the unperturbed solar wind, especially through a much higher variability of the magnetic field. Relative field variations (deviations of parameter in some interval, attributed to the average value of this interval) in magnetic layer exceed the value in solar wind in about $2-4$ times. The biggest fluctuations in the magnetic field are observed at the front shock wave and PSH regions. However, even with almost complete absence of fluctuations 
of the magnetic field in the solar wind, there are variations in magnetic layer that have high levels of disturbances. Thus, the nature of turbulent plasma flow in MSH is not directly connected to the turbulence in SW and largely is a manifestation of internal magnetosheath processes.

Furthermore, we should not forget that we analyse fluctuations of the magnetic field and not the velocity of the flow - meaning that there can be a difference between the fluid and MHD-environment.

\section{RESULTS}

During the analysis of the properties of fluctuations (absolute value) of the magnetic field module $\tau$ shift in time was chosen $(\tau)$, aliquot discrete measurements $-\tau_{\min }=0.0445 \mathrm{~s}$.

The results of magnetic field fluctuation PDF change for the solar wind plasma, foreshock region and magnetic layer region are shown in Fig. 2, 3 and 4. The evolution of magnetic field fluctuation PDF height for different time scales is shown in Fig. 5.

Exponents of the maximum magnetic field fluctuation PDF for different time scales are gathered in Table 1.

Based on plots and parameters collected in Table 1 , we can conclude that in the range of $1 \mathrm{~s}$ (except for the case of solar wind plasma), distribution significantly deviates from Gaussian. On the scale of more than $1 \mathrm{~s}$, exponent values are in the range of 0.62 to 0.47 .

An important point of the results is the presence of intermittency in the transitional areas of Earth's magnetosphere on a small scale. For solar wind plasma, the change of the maximum density function of probability is similar to a typical Gaussian distribution, in the sense that there is no intermittency.

During analysis of features of structural functions (moments of function of probability's density) of different ranks $q$ according to the time interval $\tau$ for the series of data $B(t)$, a structural function was determined through the correlation $[6,9]$ :

$$
S_{q}(\tau)=\left\langle|B(t+\tau)-B(t)|^{q}\right\rangle \sim t^{\zeta(q)},
$$

where, $\langle\ldots\rangle$ means averaging experimental data over time.

Power dependence of the type $S_{q}(\tau) \sim \tau^{\zeta(q)}$ (i. e. self-similarity - linear relation) is observed in a limited range of time scales, corresponding to the inertial range. In the transitional areas of Earth's magnetosphere such interval is observed on the scale of $\sim 1 \mathrm{~s}$ - a value that is close to ion cyclotron period for the analysed satellite measurements. Presence of data with discreteness of $22.5 \mathrm{~Hz}$ allows one to analyse structural functions of high levels and thus to determine properties of turbulence even on a small scale (up to $1 \mathrm{~s}$ ).

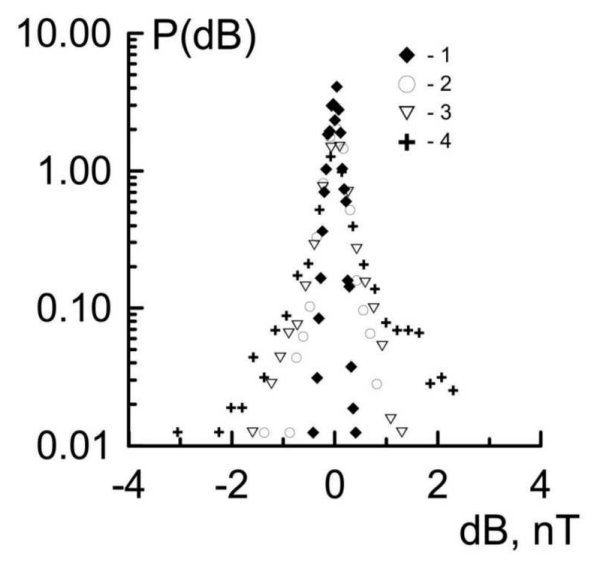

Fig. 2: Relation of absolute value of magnetic field fluctuations in solar wind PDF for Jan 6, 2007 for various time scales: where is $1-0.0445 \mathrm{~s}, 2-0.89 \mathrm{~s}, 3-1.78 \mathrm{~s}$ and $4-7 \mathrm{~s}$.

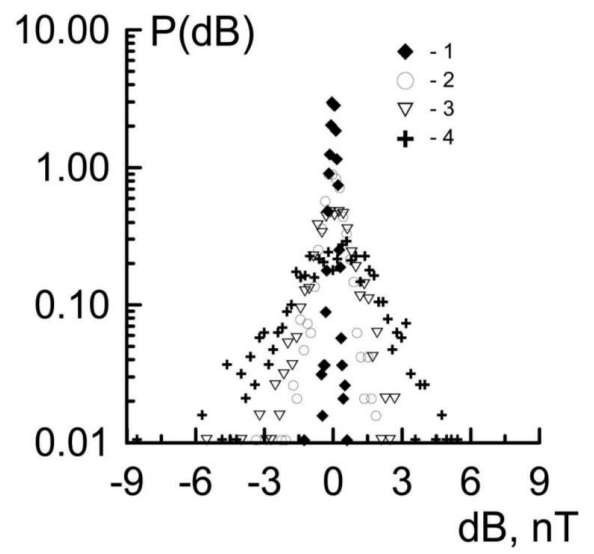

Fig. 3: Relation of absolute value of magnetic field fluctuations in foreshock region PDF for Jan 6, 2007 for various time scales: where is $1-0.0445 \mathrm{~s}, 2-0.89 \mathrm{~s}, 3$ $-1.78 \mathrm{~s}$ and $4-7 \mathrm{~s}$.

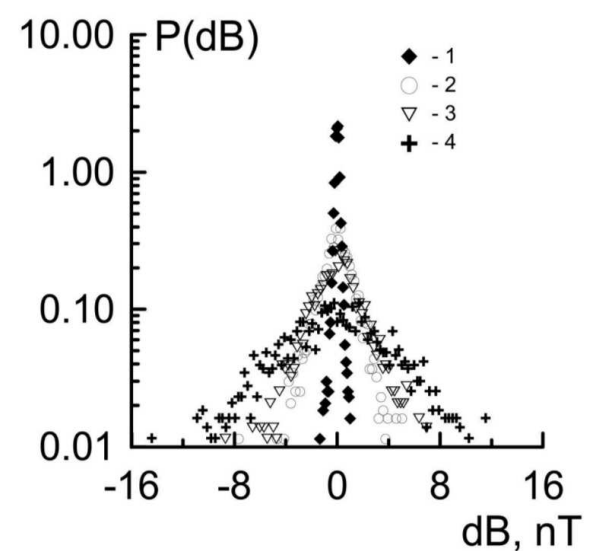

Fig. 4: Relation of absolute value of magnetic field fluctuations in magnetosheath PDF for Jan 6, 2007 for various time scales: where is $1-0.0445 \mathrm{~s}, 2-0.89 \mathrm{~s}, 3-$ $1.78 \mathrm{~s}$ and $4-7 \mathrm{~s}$. 


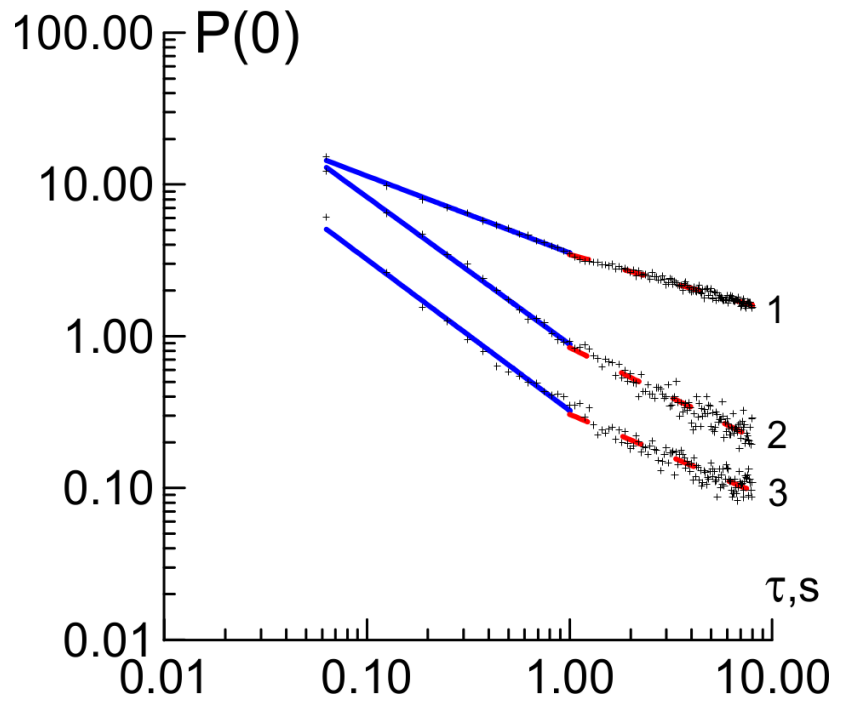

Fig. 5: Maximum value for magnetic field fluctuation PDF $P(0)$ in logarithmic scale for 3rd March 2004. Experimental data approximated with a line: $P \sim t^{-s}: 1$ - foreshock, 2 - magnetosheath, 3 - postshock. Values of $s$ presented in Table 1.

Comparison of experimental data with threedimensional models of turbulent processes was conducted by examining exponent of structural function's level, normalized to value of the third moment, since for Kolmogorov's turbulence model, K41 $\zeta(3)=3 / 3=1$.

For comparison of experimental values with twodimensional turbulence models, the dependence was found to be $\zeta(q) / \zeta(4)$ (and for Iroshnikov-Kraichnan model, it was $\zeta(4)=4 / 4=1)$.

Received dependencies $\zeta(q) / \zeta(3)$ during the analysis of small-scale turbulence and their comparison with Kolmogorov's and log-Poisson models with parameters She and Leveque (isotropic log-Poisson model $\zeta(q)=q / 9+2\left(1-(2 / 3)^{q / 3}\right.$, are shown in Fig. 6.

Comparison of experimental data with twodimensional turbulence models — IroshnikovKraichnan and Politano-Pouquet models, is shown in Fig. 7.

\section{DISCUSSION AND CONCLUSIONS}

From the analysis of the wings of the density distribution function of the probability of magnetic field fluctuations, a presence of intermittency in transitional regions of the Earth's magnetosphere was observed. Analysis of the height of the maximum function of fluctuation probability density showed that at high frequencies (on a small scale) the turbulence structure is different from that of the low frequency area. Thus, the following characteristics of turbulence can be noted: during the passage of the satellite through the plasma of the solar wind, intermittence in turbulent processes is absent (in the analysis of se- lected areas, where fluctuations of parameters are up to $0.1-0.4 \mathrm{nT}$ ). During analysis of the data for foreshock, postshock, and magnetic layer, we have two asymptotic regimes of change of the maximum function of density of probability; for large time scales change of maximum density function is similar to typical Gaussian distribution, while for small time scales observed features have non-Gaussian statistics. Intersection of two asymptotes corresponds to a value of approximately $1 \mathrm{~s}$, which is consistent with the value of the ion-cyclotron period.

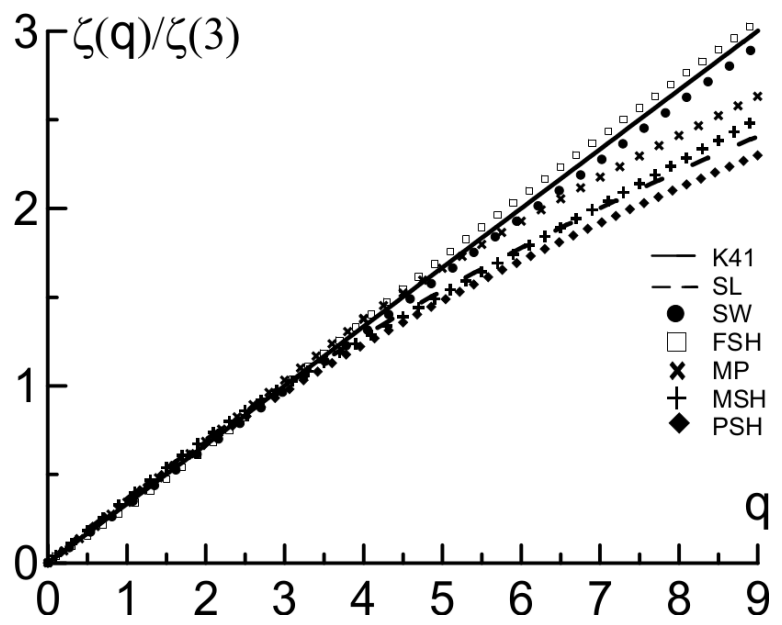

Fig. 6: Relation of exponential value of structural function of $q$-order to the 3 -rd order structural function for Jan 6, 2007. K41 — values calculated for Kolmogorov models; SL - values for isotropic log-Poisson cascade model; MP — magnetopause region; SW - solar wind region; FSH - foreshock region, MSH - magnetosheath, PSH - postshock.

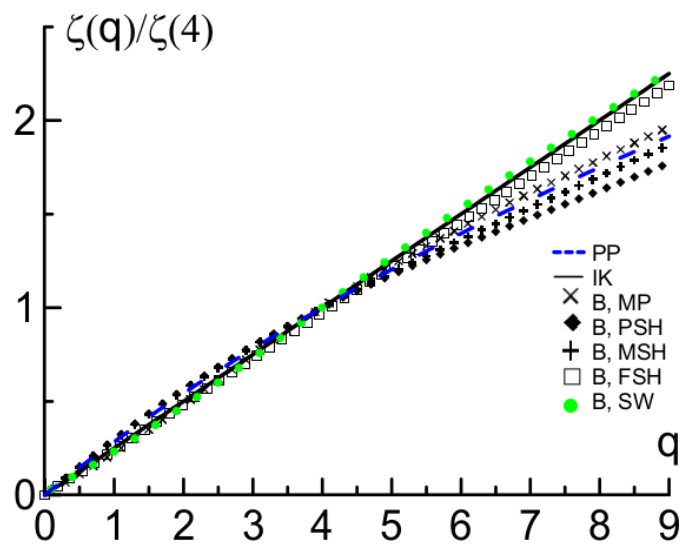

Fig. 7: Relation of exponential value of structural function of $q$-order to the 4-rd order structural function for Jan 6, 2007. IK - values calculated for IroshnikovKraichnan models; PP — values for Politano-Pouquet model; MP — magnetopause region; SW — solar wind region; FSH — foreshock region, MSH - magnetosheath, PSH - postshock. 
Having two different modes indicates two different processes: to describe processes with characteristic scales larger than the ion-cyclotron radius we can use magnetohydrodynamics equations, and to describe processes with smaller characteristic scale - kinetic equations.

It is determined that turbulent processes in solar wind plasma are close to the two-dimensional Iroshnikov-Kraichnan (in $82 \%$ of examined cases), and in the middle of magnetic layer they are well described by isotropic log-Poisson cascade model with She and Leveque parameters. The largest heterogeneity (intermittence) and anisotropy of turbulent processes is observed in the postshock region.

\section{ACKNOWLEDGEMENTS}

This work was conducted in the frame of complex program of NAS of Ukraine on space researches for 2012-2016, the grant Az. 90312 from the Volkswagen Foundation ("VW-Stiftung") and within the framework of the educational program No.2201250 "Education, Training of students, PhD students, scientific and pedagogical staff abroad" launched by the Ministry of Education and Science of Ukraine.

\section{REFERENCES}

[1] Consolini G., Kretzschmar M., Lui A. T. Y., Zimbardo G. \& Macek W. M. 2005, J. Geophys. Res., 110, A07202

[2] Dubrulle B. 1994, Phys. Rev. Lett., 73, 959

[3] Freak P. G. 1999, 'Turbulence: models and approaches', Perm National Technical University Press, Perm

[4] Frysh W. 1998, 'Turbulence: Kolmogorov heritage', Phasis, Moscow

[5] Kolmogorov A. N. 1941, Doklady Akademiia Nauk SSSR, 30, 301

[6] Kozak L. V. 2010, Space Science and Technology, 16, 1, 28

[7] KozakL. V., Pilipenko V. A., Chugunova O. M. \& Kozak P. N. 2011, Cosmic Research, 49, 194

[8] Kozak L. V., Savin S. P., Budaev V.P., Pilipenko V. A. \& Lezhen L. A. 2012, Geomagnetism and Aeronomy, 52, 445

[9] Kozak L., Lui A. \& Savin S. 2013, Odessa Astronomical Publications, 26, 268

[10] Kraichnan R. H. 1959, J. Fluid Mechanics, 5, 497

[11] Kraichnan R. H. 1970, J. Fluid Mechanics, 41, 189

[12] Politano H., Pouquet A. \& Carbone V. 1998, Europhysics Letters, 43, 516

[13] She Z.-S. \& LevequeE. 1994, Phys. Rev. Lett., 72, 336

[14] Yroshnykov P.S. 1963, AJ, 40, 742 
Table 1: Exponents of the maximum magnetic field fluctuation PDF for different time scales.

\begin{tabular}{llll}
\hline Date & Region & $S(0.00445-0.9968 \mathrm{~s})$ & $S(1-10 \mathrm{~s})$ \\
\hline $2004 / 01 / 02$ & Solar wind & $0.54 \pm 0.03$ & $0.48 \pm 0.01$ \\
& Magnetosheath & $0.97 \pm 0.034$ & $0.56 \pm 0.013$ \\
& Magnetopause & $0.77 \pm 0.031$ & $0.54 \pm 0.021$ \\
\hline $2005 / 01 / 03$ & Solar wind & $0.52 \pm 0.022$ & $0.45 \pm 0.012$ \\
& Magnetosheath & $0.87 \pm 0.023$ & $0.52 \pm 0.017$ \\
& Magnetopause & $0.64 \pm 0.032$ & $0.5 \pm 0.015$ \\
\hline $2005 / 05 / 03$ & Solar wind & $0.50 \pm 0.027$ & $0.46 \pm 0.019$ \\
& Magnetosheath & $0.98 \pm 0.036$ & $0.52 \pm 0.022$ \\
& Magnetopause & $0.64 \pm 0.05$ & $0.53 \pm 0.031$ \\
\hline $2006 / 05 / 04$ & Solar wind & $0.5 \pm 0.03$ & $0.49 \pm 0.014$ \\
& Foreshock & $0.77 \pm 0.034$ & $0.46 \pm 0.014$ \\
& Magnetosheath & $0.91 \pm 0.042$ & $0.6 \pm 0.017$ \\
\hline $2007 / 03 / 20$ & Solar wind & $0.54 \pm 0.041$ & $0.43 \pm 0.012$ \\
& Magnetosheath & $0.87 \pm 0.072$ & $0.51 \pm 0.021$ \\
& Magnetopause & $0.76 \pm 0.051$ & $0.53 \pm 0.015$ \\
\hline $2007 / 01 / 06$ & Solar wind & $0.52 \pm 0.06$ & $0.48 \pm 0.018$ \\
& Foreshock & $0.87 \pm 0.083$ & $0.57 \pm 0.028$ \\
& Postshock & $0.99 \pm 0.091$ & $0.58 \pm 0.031$ \\
& Magnetosheath & $0.82 \pm 0.097$ & $0.57 \pm 0.034$ \\
& Magnetopause & $0.79 \pm 0.044$ & $0.43 \pm 0.031$ \\
\hline $2007 / 04 / 02$ & Solar wind & $0.51 \pm 0.052$ & $0.49 \pm 0.016$ \\
& Foreshock & $0.86 \pm 0.058$ & $0.5 \pm 0.013$ \\
& Magnetosheath & $0.93 \pm 0.092$ & $0.53 \pm 0.045$ \\
\hline $2007 / 03 / 20$ & Solar wind & $0.54 \pm 0.083$ & $0.43 \pm 0.019$ \\
& Magnetosheath & $0.87 \pm 0.099$ & $0.51 \pm 0.045$ \\
& Magnetopause & $0.76 \pm 0.097$ & $0.53 \pm 0.028$ \\
\hline
\end{tabular}

